\title{
Computational inteligence in optimization of machining operation parameters of ST-37 steel
}

\begin{abstract}
Optimal selection of cutting parameters is one of the significant issues in achieving high quality machining. In this study, a method for the selection of optimal cutting parameters during lathe operation is presented. The present study focuses on multiple-performance optimization on machining characteristics of St-37 steel. The cutting parameters used in this experimental study include cutting speed, feed rate, depth of cut and rake angle. Two output parameters, namely, surface roughness and tool life are considered as process performance. A statistical model based on linear polynomial equations is developed to describe different responses. For optimal conditions, the Non-dominated Sorting Genetic Algorithm (NSGA) is employed in achieving appropriate models. The optimization procedure shows that the proposed method has a high performance in problem-solving.
\end{abstract}

Keyword: Non-dominated; Optimization; Sorting genetic algorithm; Statistical model; Surface roughness; Tool life 